\title{
$\mathrm{GPU}$ 를 이용한 기타의 음 합성을 위한 효과적인 병렬 구현
}

\author{
강성모*, 김종면*
}

\section{An Effective Parallel Implementation of Sound Synthesis of Guitar using GPU}

Sung-Mo Kang, Jong-Myon Kim

\section{요 약}

본 논문에서는 $\mathrm{GPU}$ 환경에서 기타의 음 합성을 위한 물리적 모델링의 효율적인 병렬 구현 방법을 제안한다. 물 리적 모델링을 이용하여 기타의 개방현(E2, A2, D3, G4, B3, E4)들의 기본음을 합성하기 위해 각 개방현 음 합 성을 위한 적절한 필터 계수를 사용하였고, 지연 라인의 길이를 조절하였다. 또한 물리적 모델링 알고리즘을 분석 한 결과 지연 라인의 길이만큼 병렬성을 갖는 것을 확인하였다. 따라서 각 개방현의 기타 음을 합성하기 위해 지연 라인의 길이만큼 CUDA 코어를 할당한 후 최적의 성능을 보이도록 알고리즘을 병렬 구현하였다. 모의실험결과, GPU를 이용하여 합성한 기타 음과 원음과의 스펙트럼이 매우 유사하였고, GPU는 기존 고성능 TI DSP보다 68 배, CPU보다 3배의 성능 향상을 보였다. 또한, 본 논문에서는 물리적 모델링 알고리즘을 멀티 GPU시스템에서도 구현하고 성능을 분석하였다.

- Keywords : 물리적 모델링 합성, 기타 음, 병렬 구현, 그래픽 처리 유닛, OpenCL

\begin{abstract}
This paper proposes an effective parallel implementation of a physical modeling synthesis of guitar on the GPU environment. We used appropriate filter coefficients and adjusted the length of delay line for each open string to generate 44,100 six-polyphonic guitar sounds (E2, A2, D3, G4, B3, E4) by using physical modeling synthesis. In addition, we analyzed the physical modeling synthesis algorithm and observed that we can exploit parallelism inherent in the length of delay
\end{abstract}

-제 1 저자 : 강성모 •교신저자 : 김종면

•투고일 : 2013. 2. 27, 심사일 : 2012. 3. 23, 게재확정일 : 2013. 3. 29.

* 울산대학교 전기공학부 (School of Electrical Engineering, University of Ulsan)

※ 이 논문은 2012년 제46차 한국컴퓨터정보학회 하계학술대회에서 우수논문상을 수상한 논문(“물리적 모델링을 통한 실시간 기타 음 합성의 GPU 구현")을 확장한 것임

※ 이 논문은 2013 년도 정부(미래창조과학부)의 재원으로 한국연구재단의 지원을 받아 수행된 연구임 (No. NRF-2013R1A2A2A05004566). 
line. Thus, we assigned CUDA cores as many as the length of delay line and effectively implemented the physical modeling synthesis using GPU to achieve the highest performance. Experimental results indicated that synthetic guitar sounds using GPU were very similar to the original sounds when we compared their spectra. In addition, GPU achieved $68 \mathrm{x}$ and $3 \mathrm{x}$ better performance than high-performance TI DSP and CPU, respectively. Furthermore, this paper implemented and evaluated the performance of multi-GPU systems for the physical modeling algorithm.

- Keywords: Physical modeling synthesis, guitar sound, parallel implementation, graphics processing unit, OpenCL

\section{I. 서 론}

최근 디지털 신호처리 기술과 음향 기술의 발전으로 직접 악기를 연주하지 않고 디지털 신호를 합성하여 오디오 신호를 만들어 내는 전자악기들이 개발되어오고 있다[1-3]. 초기의 전자악기들은 음 합성 시 녹음 된 악기의 음을 변조하여 새로 운 음을 생성하는 방식을 주로 사용하지만 아날로그 악기의 부드러운 음색을 충분히 재현하지 못하는 단점을 가지고 있어 이를 보완하기 위한 다양한 음 합성 기법들이 연구되고 있다. 대표적인 음 합성 방법으로는 샘플링 합성, 가산 합성, 감산 합성, 주파수 변조 합성, 스펙트럼 모델링 합성, 물리적 모델 링 합성 기법 등이 있다. 그 중 물리적 모델링 기반의 음 합성 방법은 고음질의 자연스러운 음을 합성할 수 있어 현악기를 위한 음 합성 알고리즘으로 적합하다[4]. 하지만 물리적 모델 링 기반의 음 합성은 많은 양의 산술 연산이 요구되므로 실시 간 출력을 만족시키는데 제약을 가진다. 이러한 많은 연산량 을 요구하는 멀티미디어 애플리케이션을 위해 GPU와 같은 가속기들의 사용이 증가하고 있다[5].

GPU는 2D 및 3D 그래픽 연산을 처리하기 위한 목적으로 개발된 그래픽 연산전용의 프로세서이다. 오늘날의 GPU는 그 래픽 전용 연산장치의 목적뿐만 아니라 산술연산에서 발생하는 작업부하를 줄이기 위한 범용 연산장치로도 사용되며 이를 GPGPU(General-Purpose computing on Graphics Processing Units)라고 부른다. GPU는 높은 부동 소수점 연 산속도와 메모리 대역폭 덕분에 다양한 과학 및 공학 계산을 가속화 할 수 있다 $[6,7]$. 이러한 $\mathrm{GPU}$ 프로그래밍은 단순한 산술 반복 연산을 프로세서에 적재된 많은 수의 코어를 통해 효과적으로 병렬 처리할 수 있어 고성능 컴퓨팅에 있어서 가장
적합한 솔루션이라고 평가되고 있다. 이러한 GPU를 범용의 목 적으로 사용하기위해서는 OpenCL(Open Computing Language)과 같은 대규모 병렬 프로그래밍 프레임워크가 필 요하다. OpenCL은 GPU의 제조사나 하드웨어 아키텍처에서 상관없이 GPU 프로그래밍이 가능하여 GPU 프로그래밍의 유 연성을 높이고 광범위한 사용을 가능하게 한다[8].

본 논문에서는 물리적 모델링 기반의 기타 음 합성을 $\mathrm{GPU}$ 상에서 병렬처리 될 수 있도록 OpenCL을 사용하여 알 고리즘을 병렬 구현한다. 인텔 Xeon(R) x5690 @ $3.47 \mathrm{GHz}$ 와 NVIDIA Geforce GTX 580 상에서 샘플링율 이 $44.1 \mathrm{kMz}, 16$ 비트의 양자화 데이터 음을 합성하였으며, 합성음은 원음과의 스펙트럼 비교 결과 원음과 매우 유사함을 확인할 수 있었다. 또한 제안한 방법의 성능 평가를 위해 TI $\mathrm{DSP}$ 와 실행 시간 측면에서 성능을 비교하여 좋은 결과를 보 였다.

본 논문의 구성은 다음과 같다. 2장에서는 실험에 사용되 는 GPU와 병렬처리 프레임워크인 OpenCL에 대해 설명한 다. 3 장에서는 물리적 모델링 알고리즘에 대해 설명하고 이에 대한 병렬처리 방법에 대해서 설명한다. 4장에서는 실험환경 및 음 합성 결과를 보이고, 제안한 병렬 구현 방법과 사용 프 로세서의 실행 시간 측면에서 성능을 분석한다. 마지막으로 5 장에서 본 논문의 결론을 맺는다.

\section{II. 관련 연구}

\section{GPU}

본 논문에서 사용된 GPU 프로세서는 NVIDIA사의 GeForce GTX 580으로, GTX 580 아키텍처는 그림 1과 같 
이 16개의 스트리밍 멀티프로세서 (streaming multiprocessor, $\mathrm{SM}$ )를 가진다. SM은 명령어를 인출하여 전체 스트리밍 프로세서(streaming processor, $\mathrm{SP}$ )에 전송 하고, 각 $\mathrm{SP}$ 들은 명령어를 동시에 수행한다. 하나의 $\mathrm{SM}$ 은 다음과 같은 특징을 갖는다[9].

- FPU(floating point unit)와 ALU(arithmetic logic unit)로 구성된 32개의 CUDA Core 또는 SP

- 메모리로부터 읽기/쓰기를 실행하는 16 개의 $\mathrm{LD} / \mathrm{ST}$ (lode/store unit)

- 초월함수를 처리하는 $\mathrm{SFU}$ (special function unit)

- Warp라 불리는 병렬스레드 그룹을 관리하는 Warp scheduler

- $48 \mathrm{~KB}$ 의 공유 메모리와 L1 캐시(cache)

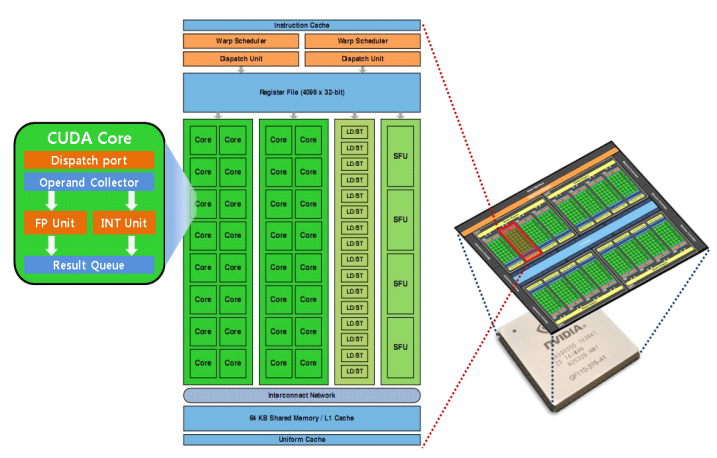

그림 1. GeForce GTX 580 구조

Fig. 1. GeForce GTX 580 Architecture

$\mathrm{SM}$ 내의 32개의 SP들은 공유 메모리(shared memory) 를 통해 데이터를 공유할 수 있으며, $\mathrm{SM}$ 들은 전역 메모리 (global memory)를 통해 데이터를 공유한다.

\section{OpenCL}

OpenCL은 애플, AMD, 인텔, NVIDIA, 삼성, NOKIA 등의 프로세서 업체, 시스템 $\mathrm{OEM}$ 업체, 응용 프로그램 업체 및 다양한 업계가 참여하여 표준화 작업을 진행한 개방형 범용 병렬 컴퓨팅 프레임워크이다. OpenCL은 CUDA(Compute Unified Device Architecture)와 달리 여러 제조업체의 GPU를 단일 인터페이스를 통해 지원하는 장점이 있다. OpenCL은 여러 개의 스레드(thread)에서 각기 다른 데이터 를 사용하여 병렬적으로 실행되는 SIMT(single instruction multiple thread) 방식으로 동작한다.

그림 2는 OpenCL에서의 $\mathrm{ND}$ (N차원)-Range의 구성을
나타낸다. 워크아이템(work-item)은 CUDA 스레드와 같은 의미이며 가장 작은 단위의 실행객체이다. 각각의 워크아이템 은 동일한 커널 함수를 실행하여 같은 코드로 동작한다. 워크 그룹(work-group)은 CUDA thread block과 같은 의미이 며, 워크아이템들끼리 동기화가 가능하여 협력 작업을 수행할 수 있다. ND-Range는 워크그룹들로 구성된다.

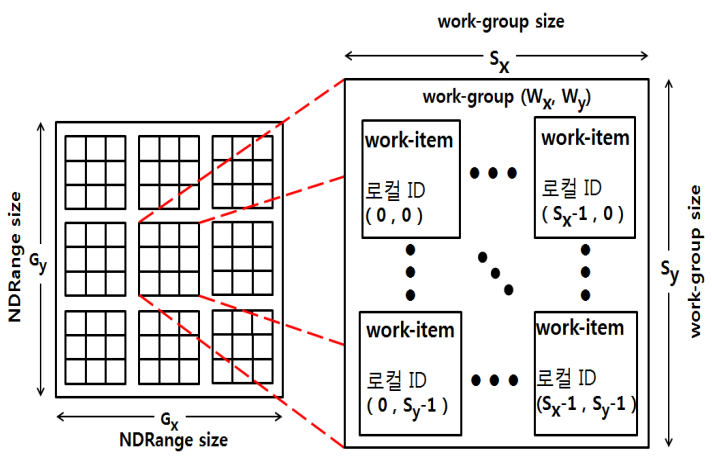

그림 2. ND-Range 구조

Fig. 2. ND-Range architecture

\section{III. 본 론}

\section{1. 물리적 모델링 합성}

물리적 모델링 합성은 악기가 음을 생성하는 물리적인 현 상을 수식적으로 표현한 것이다. 물리적 모델링 합성의 많은 계산량으로 인해 기존에는 음 합성을 위해 해당 음을 녹음하 여 재현하는 샘플링 방법이 주로 사용되어왔다. 샘플링 합성 의 경우는 전자악기 혹은 스마트 기기와 연동하여 다양한 악 기를 연주할 경우 많은 양의 녹음된 음 정보가 요구되어 하드 웨어적인 제약을 갖는다. 반면 $\mathrm{GPU}$ 와 같은 고성능 프로세싱 유닛과 물리적 모델링 합성을 이용할 경우 적은양의 여기 신 호와 필터 계수를 이용하여 보다 풍부하고 자연스러운 음 합 성이 실시간으로 가능하다. 그림 3은 물리적 모델링을 합성에 기반을 둔 현 모델을 나타낸다.

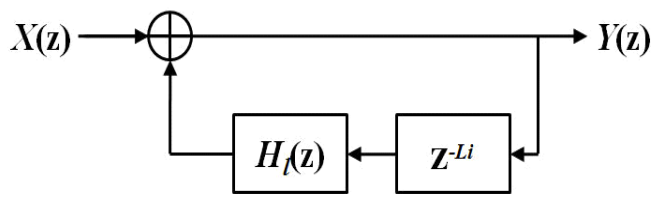

그림 3. 기타의 현 모델 블록 다이어그램

Fig. 3. A block diagram of the string model of Guitar 
그림 3에서 X(z)는 현 모델의 입력으로 기타 현의 특성을 제외한 기타 몸체(guitar body)와 브릿지(guitar bridge) 등의 특성을 포함한 여기 신호(excitation signal)이다. Z-Li는 지연 라인(delay line)을 나타내며 지연 라인의 길이 $\mathrm{L}$ 은 합성음의 샘플링율 $f_{s}$ 과 합성음의 기본 주파수 $f_{0}$ 로 식 (1)과 같이 계산된다.

$$
L=f_{s} / f_{0} .
$$

현 진동의 감쇄현상을 표현하기 위한 루프 필터(loop filter) $H_{l}(z)$ 는 저역 통과 필터(low-pass filter)의 특성 을 가지며 식 (2)과 같이 표현된다[10].

$$
H_{l}(z)=g \frac{1+a_{1}}{1+a_{1} z^{-1}},
$$

여기서 $g$ 는 $0 \mathrm{~Hz}$ 에서의 필터 이득, $a_{1}$ 은 차단 주파수를 결정하는 필터 계수이다.

\section{2. 물리적 모델링 합성의 병렬 구현}

기타 음 합성을 위한 물리적 모델링 합성은 그림 3에서와

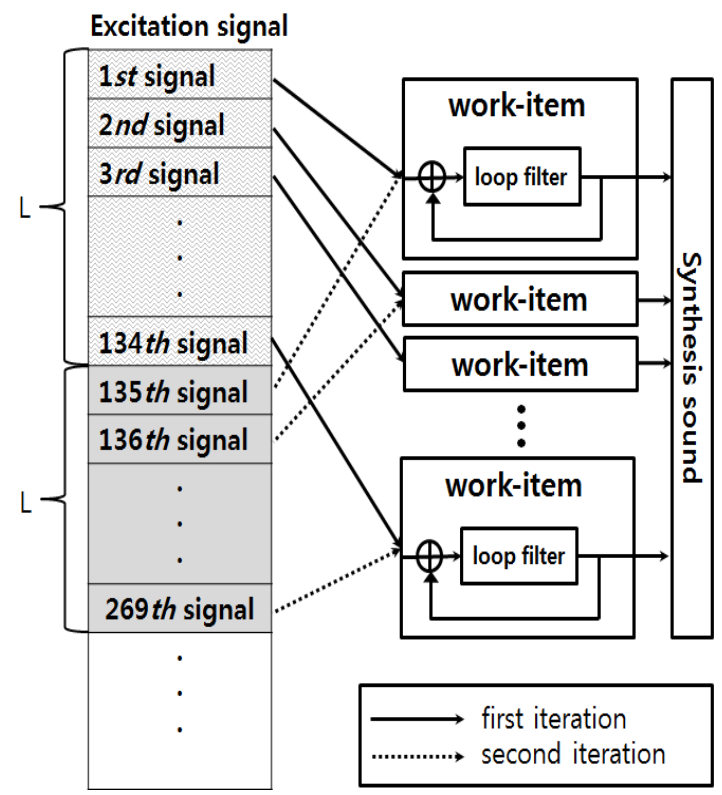

그림 4. 기타의 현 모델 병렬 구현

Fig. 4. A parallel implementation of the string model of Guitar
같이 여기 신호 $X(\mathrm{z})$ 가 입력으로 들어오면 지연 라인을 거쳐 서 루프 필터를 통과하게 되고, 이와 같은 과정이 반복되어 최종 음이 합성된다. 즉 이전의 연산 결과는 지연 라인을 통 과 한 후 음 합성을 위한 입력으로 다시 사용되는 데이터 종 속성을 갖게 된다. 반면 지연 라인 Z-L에서의 데이터는 데이 터 종속성을 갖지 않으므로 지연 라인의 길이만큼 병렬 구현 이 가능하다.

그림 4 는 기타 개방현 1 번 $(\mathrm{E} 4,329.63 \mathrm{~Hz})$ 음 합성을 위 한 병렬 구현 예이다. 먼저 개방현 1 번의 병렬 구현을 위해 데 이터 독립성을 갖는 지연길이 $\mathrm{L}(44,100 / 329.63 \risingdotseq 133)$ 만큼의 워크 아이템을 할당한다. 이때 각 워크 아이템에서 하나의 음 샘플(sample)을 합성하여 한 번에 지연길이 L(133)만큼의 샘플이 동시에 합성된다. 이를 GPU 상에서 맵핑하면 그림 5 와 같이 나타낼 수 있다. 하나의 여기 신호 샘플을 하나의 $\mathrm{CUDA}$ 코어에 할당 되도록 하여 지연 라인의 길이만큼 CUDA 코어에 맵핑한다. 하나의 SM마다 19개의 CUDA 코 어를 사용하여 7 개의 $\mathrm{SM}$ 이 할당되어 한번에 133 개의 샘플이 합성된다. 즉 1 초 길이의 기타 음 합성을 위해서는 이와 같은 과정을 332 번 반복하여 총 44,156 개의 샘플을 합성한다.

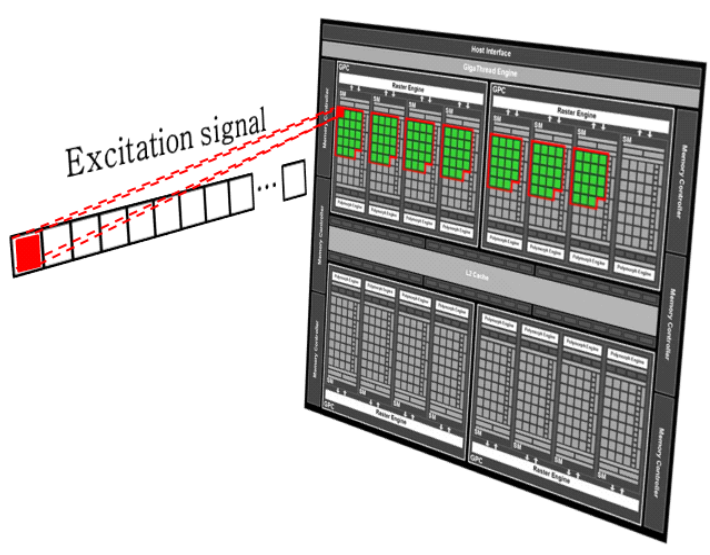

그림 5. 기타의 1번 현에 대한 워크 아이템의 GPU 맵핑

Fig. 5. GPU Mapping of work-item on 1st string of guitar

\section{IV. 실험 결과}

\section{1. 실험 환경}

본 실험에서 사용된 $\mathrm{CPU}$ 는 인텔사의 Xeon x5690이며, 연산에 사용된 GPU는 NVIDIA의 GeForce GTX 580이다. 표 1은 물리적 모델링 합성을 이용한 기타 음 합성을 위해 사 

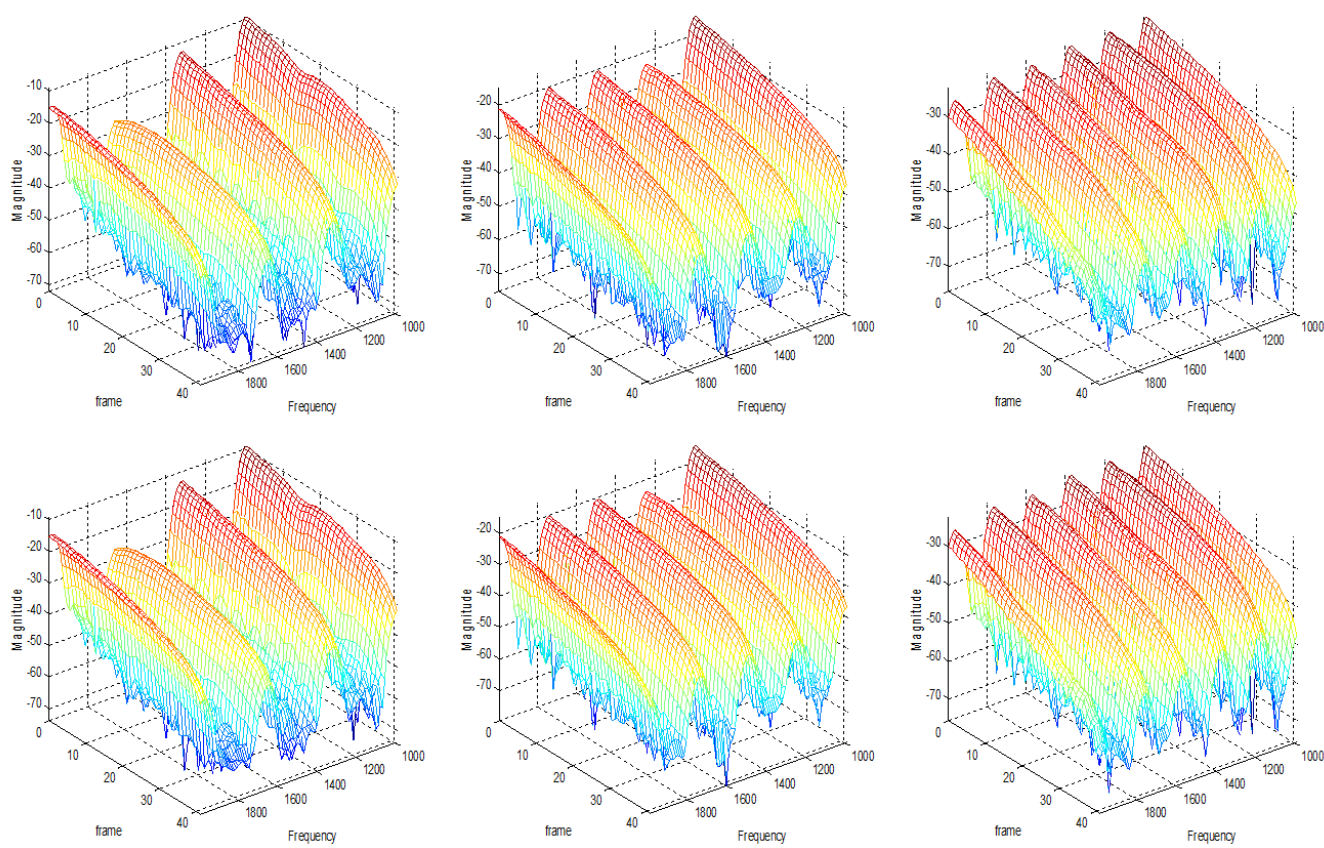

(a)

(b)

(c)
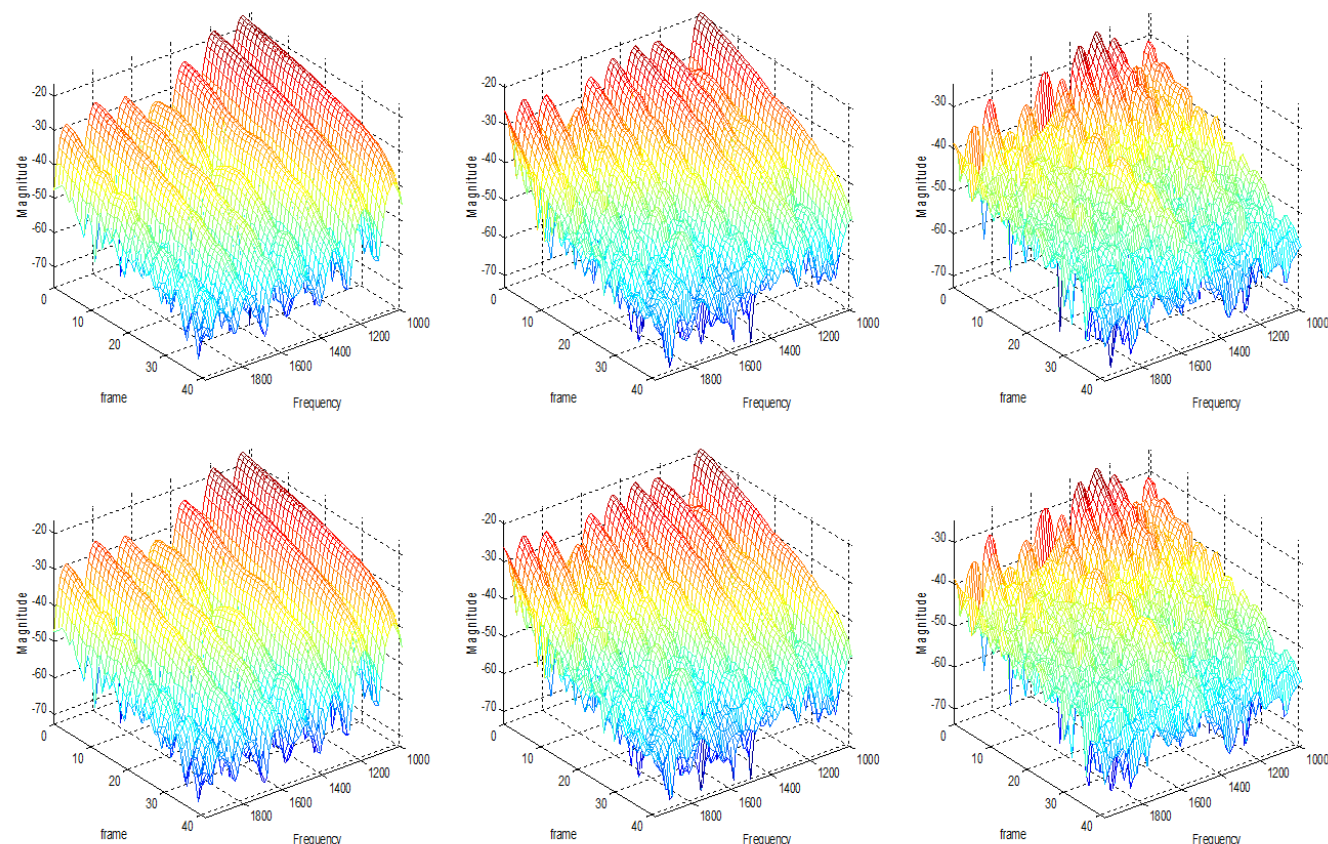

(d)

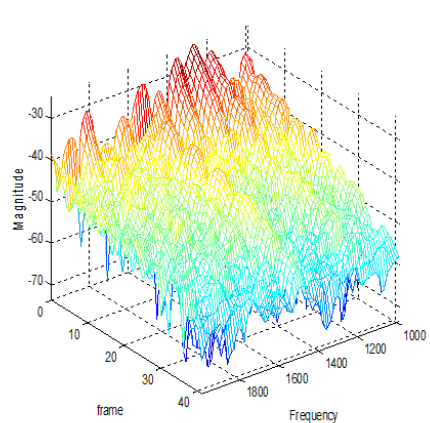

(e)

(f)

그림 6.악기의 원음(상)과 GPU를 이용한 합성 음(하)에 대한 6가지 현의 스펙트럼 : (a) 1번 현, (b) 2번 현, (c) 3번 현, (d) 4번현, (e) 5번 현, (f) 6번 현

Fig. 6. Spectra of six strings for original sounds(top) and synthesized sounds(bottom) using GPU : (a) 1st string, (b) 2nd string, (c) 3rd string, (d) 4th string, (e) 5th string, (f) 6th string 
용된 전체 시스템의 실험 환경을 보여준다. 사용된 GPU는 512 개의 CUDA 코어를 가지고 있으며 GPU 장치의 자세한 사양은 표 2 와 같다.

표 1. 시스템 환경

Table 1. System Environment

\begin{tabular}{|c|c|}
\hline 실험 요소 & 장치 명 \\
\hline $\mathrm{CPU}$ (수) & Intel Xeon $\times 56903.46 \mathrm{GHz}$ (2) \\
\hline Memory & 144 (GB) \\
\hline OS & CentOS 5.8 \\
\hline GPU & NVIDIA GeForce GTX 580 \\
\hline
\end{tabular}

표 2. NVIDIA GeForce GTX 580 사양

Table 2. Specification of the NVIDIA GeForce GTX 580

\begin{tabular}{|c|c|}
\hline 항목 & 값 \\
\hline Global memory & $3 \mathrm{~GB}$ \\
\hline local memory & $48 \mathrm{~KB}$ \\
\hline Clock Frequency & $1.51 \mathrm{GHz}$ \\
\hline Max workGroup & 1024 \\
\hline Max Compute Units & 16 \\
\hline CUDA version & 4.2 .1 \\
\hline OpenCL version & 1.1 \\
\hline
\end{tabular}

\section{2. 음 합성 결과}

그림 6 은 본 논문에서 제안한 물리적 모델링 합성의 병렬 구현 방법을 이용하여 합성한 개방현 음과 원음과의 스펙트럼 비교 결과를 보여준다. 그림 6 에서 보는 바와 같이 합성음과 원음의 스펙트럼은 매우 유사한 결과를 보였고, 청취 결과에 서도 거의 동일한 결과를 보였다. 합성한 음은 아래 웹사이트 를 통해 확인할 수 있다.

http://eucs.ulsan.ac.kr/KSCI/2013/01/GPU-PM.

\section{3. $\mathrm{CPU}$ 와 GPU간의 성능 비교}

$\mathrm{GPU}$ 를 이용한 기타 음 합성 알고리즘의 성능을 평가하기 위해 $\mathrm{GPU}$ 와 $\mathrm{CPU}$ 의 성능을 비교하였다. 그림 7은 $\mathrm{CPU}$ 와 $\mathrm{GPU}$ 에서 6 개 현의 음을 각각 합성하는데 걸리는 시간을 보 여준다. 순차처리를 하는 $\mathrm{CPU}$ 는 6 개의 현에 대해 같은 수준 의 시간을 소비한다. 하지만 병렬처리 알고리즘을 GPU에서 수행하게 되면 각 현의 지연길이 $\mathrm{E} 4(133), \mathrm{B} 3(178)$, $\mathrm{G} 3(226), \mathrm{D} 3(300), \mathrm{A} 2(402), \mathrm{E} 2(535)$ 에 따라 병렬성이 달라지고, 병렬성이 높은 현의 실행시간은 낮은 현에 비해 실 행시간이 더 빠른 것을 알 수 있다.

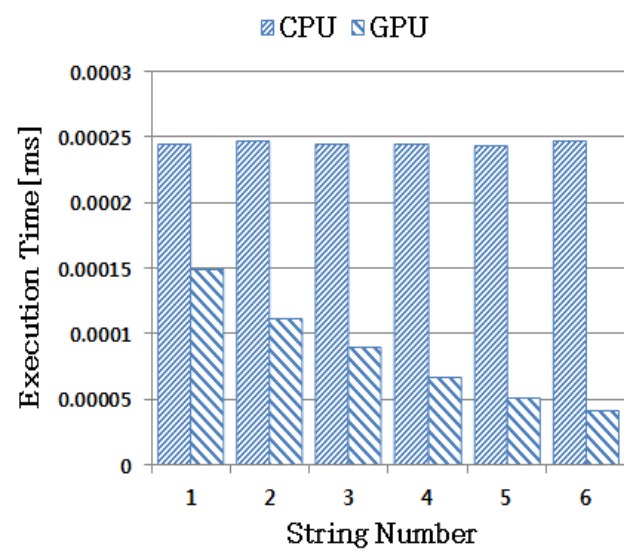

그림 7. 기타의 각 현에 대한 합성시간

Fig. 7. Synthesis Time for each string on guitar

본 논문에서는 1 개의 GPU와 $\mathrm{CPU}$ 간의 성능 비교 뿐만 아니라 다수의 GPU를 사용하였을 경우 음 합성 알고리즘의 성능 추이 변화도 분석하였다. 이러한 다수의 GPU 환경에서 성능을 분석하기 위해 NVIDIA에서 제공하는 Visual Profiler을 이용하였다. Visual Profiler는 CUDA나 OpenCL 실행 파일을 실행하여 타임라인을 통하여 GPU상 에서 알고리즘의 성능 분석과 병목 현상에 대한 정보를 볼 수 있는 크로스 플랫폼 성능 프로파일링 도구이다[11]. Visual Profiler를 이용하여 여기신호를 호스트(Host)에서 디바이 스(Device)로 전송하는데 필요한 시간 $\mathrm{HtoD}$, 합성 음을 디 바이스에서 호스트로 읽어오는데 필요한 시간 $\mathrm{DtoH}$, 실제 음 합성에 사용되는 시간을 측정하였다.

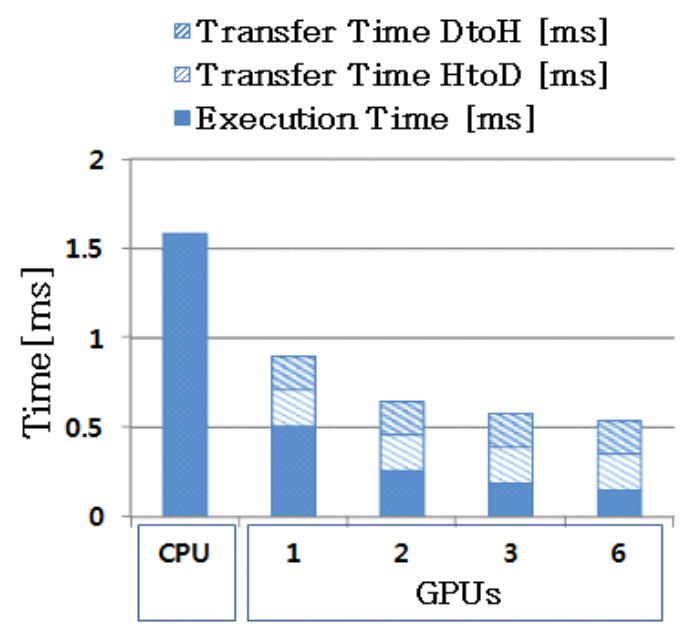

그림 8. 멀티 GPU의 성능 비교

Fig. 8. Performance comparison between multi-GPU 


\section{4. 상용 프로세서와의 성능 비교}

본 논문에서는 네 가지의 형태의 GPU 시스템에서 기타 음 합성 알고리즘의 성능을 평가하였을 뿐만아니라 기존 고성 능 TI DSP(TI TMS320C6416)와 CPU(Xeon x5690)와 의 성능도 비교하였다. 표 3 은 $\mathrm{GPU}$ 장치의 수 $(1,2,3,6)$ 를 변화시키며 측정한 성능을 상용 프로세서들과 비교한 결과 를 보여준다. 성능 비교 결과, 신호처리 분야에서 많이 사용 되는 TI DSP보다 최소 68 배에서 최대 232배 높은 성능을 보였으며, $\mathrm{CPU}$ 보다 최소 3 배에서 최대 10 배 높은 성능 향상 을 보였다.

표 3. 상용 프로세서 이키텍처 간의 성능 비교 Table 3. Performance comparison between commercial processor architectures

\begin{tabular}{|c|c|c|c|c|c|c|}
\hline 항목 & $\begin{array}{c}\text { TI } \\
\text { DSP }\end{array}$ & CPU & \multicolumn{4}{|c|}{$\mathrm{GPU}$} \\
\hline $\begin{array}{c}\text { Number } \\
\text { of Device }\end{array}$ & 1 & 1 & 1 & 2 & 3 & 6 \\
\hline Core & 1 & 6 & 512 & $\begin{array}{c}102 \\
4\end{array}$ & $\begin{array}{c}153 \\
6\end{array}$ & $\begin{array}{c}307 \\
2\end{array}$ \\
\hline $\begin{array}{c}\text { Clock } \\
\text { Frequency }\end{array}$ & $\begin{array}{c}720 \\
\mathrm{MHz}\end{array}$ & $\begin{array}{c}\mathrm{GHz} \\
\mathrm{GH}\end{array}$ & $\begin{array}{c}1.51 \\
\mathrm{GHz}\end{array}$ & $\begin{array}{c}1.51 \\
\mathrm{GHz}\end{array}$ & $\begin{array}{c}1.51 \\
\mathrm{GHz}\end{array}$ & $\begin{array}{c}1.51 \\
\mathrm{GHz}\end{array}$ \\
\hline $\begin{array}{c}\text { Execution } \\
\text { Time[ms] }\end{array}$ & 34.5 & 1.58 & 0.90 & 0.64 & 0.58 & 0.54 \\
\hline
\end{tabular}

\section{V. 결 론}

본 논문에서는 물리적 모델링 기반의 기타 음 합성 알고리 즘을 병렬화하였으며, OpenCL을 사용하여 GPU 상에서 효 율적으로 실행되도록 맵핑(mapping)하였다. 병렬 구현된 알 고리즘의 결과를 확인하기 위해 $44.1 \mathrm{kHz}$ 의 샘플링율로 합성 된 음과 원음의 청취 및 스펙트럼 분석 결과 거의 동일함을 알 수 있었다. 또한 음 합성에 사용되는 GPU의 수를 변화시 키며 실험한 결과 GPU가 1개에서 2개로 늘어날 때 실행시간 은 약 1.97 배, 3 개일 때 약 2.69 배, 6 개일 때 약 3.4 배의 성 능 항상을 보였다. 하지만 데이터 전송을 위한 시간을 포함한 총 실행시간은 2 개 일 때 약 1.39 배, 3 개일 때 약 1.55 배, 약 1.67 배의 성능 항상을 보였다. 또한 1개의 GPU를 사용 한 기타 음 합성은 TI DSP보다 실행시간에서 약 68 배 높은 성능을 보였으며, $\mathrm{CPU}$ 보다는 약 3 배의 성능 향상을 보였다. 향후에는 기타 음 합성 알고리즘을 위해 성능 및 에너지 효율 측면에서 $\mathrm{GPU}$ 내 최적의 코어 개수를 결정하는 연구를 수 행할 예정이다.

\section{참고문헌}

[1] M. karjalainen, T.Maki-Patola, A. Kanerva, A. Huovilainen, and P. janis, "Virtual air guitar," in Proc. of AES Convention, pp. 2-19, Oct. 2004.

[2] L. Kessous, J. Castet, and D. Arfib, "GXtar, an interface using guitar techniques," in Proc. of International Conference on New Interfaces for Musical Expression, pp. 192-195, June 2006.

[3] J. Kanebako, J. Gibson, and L. Mignonneau, "Mountain guitar : a musical instrument for everyone," in Proc. of International Conference on New Interfaces for Musical Expression, pp. 396-397, June 2007.

[4] M. Kang, S. Cho, and U. Chong, "Implementation of Non-Stringed Guitar Based on Physical Modeling Synthesis," Journal of the Acoustical Society of Korea, Vol. 28, No. 8, pp.119-126, Feb. 2009.

[5] J. E .Stone, D. Gohara, G. Shi, "OpenCL : A parallel programming standard for heterogeneous computing systems", Computing in Science and Eng, Vol. 12, No. 3, pp. 66-73, May 2010.

[6] H.-G. Jeon, J.-W. Ahn, J.-M. Kim, and C.-H. Kim, "Memory Delay Comparison between 2D GPU and 3D GPU," Journal of The Korea Society of Computer and Information, Vol. 17, No. 7, pp. 1-11, July 2012.

[7] H.-J. Choi, S.-G. Kang, J.-M. Kim, and C.-H. Kim, "Analysis of the CPU/GPU Temperature and Energy Efficiency depending on Executed Applications," Journal of The Korea Society of Computer and Information, Vol. 17, No. 5, pp. 9-19. May 2012.

[8] O. E. Albayrak, I. Akturk, O. Ozturk, "Effective Kernel Mapping for OpenCL Applications in Heterogeneous Platforms," Proc. of International Conference on Parallel Processing Workshop, pp. 81-88, Sept. 2012.

[9] NVIDIA Fermi Compute Architecture Whitepaper, http://www.nvidia.com/content/PDF/fermi 
_white_papers/NVIDIA_Fermi_Compute_Architec ture_Whitepaper.pdf

〔10] V. Valimaki, J. Juopaniemi, M. Karjalainen, and Z. Janosy, "Physical Modeling of Plucked String Instruments with Application to Real-time Sound Synthesis," J. Audio Eng. Soc., Vol. 44, No. 5, pp. 331-353, May 1996.

[11] NVIDIA Visual Profiler, https://developer .nvidia .com/nvidia-visual-profiler

\section{저 자 소 개}

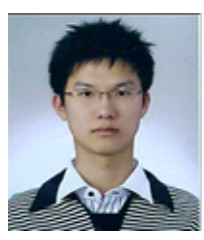

강 성 모

2011: 울산대학교

컴퓨터정보통신공학부 공학사.

현 재: 울산대학교

전기공학부 석사과정.

관심분야: 멀티미디어 신호처리,

임베디드 소프트웨어,

$\mathrm{SoC}$ 설계

Email : dryujinwolf@mail.ulsan.ac.kr

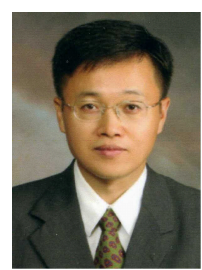

김 종 면

1989: 명지대학교 전기공학과 공학사.

2000: University of Florida

전기컴퓨터공학과 공학석사.

2004: Georgia Tech.

전기컴퓨터공학과 공학박사.

현 재: 울산대학교 전기공학부 교수

관심분야: 컴퓨터구조, 병렬프로세서,

멀티미디어 신호처리,

$\mathrm{SoC}$ 설계

Email : jongmyon.kim@gmail.com 